\title{
ON UNIQUENESS FOR TIME HARMONIC ANISOTROPIC MAXWELL'S EQUATIONS WITH PIECEWISE REGULAR COEFFICIENTS
}

\author{
JOHN M. BALL, YVES CAPDEBOSCQ, AND BASANG TSERING XIAO
}

\begin{abstract}
We are interested in the uniqueness of solutions to Maxwell's equations when the magnetic permeability $\mu$ and the permittivity $\varepsilon$ are symmetric positive definite matrix-valued functions in $\mathbb{R}^{3}$. We show that a unique continuation result for globally $W^{1, \infty}$ coefficients in a smooth, bounded domain, allows one to prove that the solution is unique in the case of coefficients which are piecewise $W^{1, \infty}$ with respect to a suitable countable collection of sub-domains with $C^{0}$ boundaries. Such suitable collections include any bounded finite collection. The proof relies on a general argument, not specific to Maxwell's equations. This result is then extended to the case when within these sub-domains the permeability and permittivity are only $L^{\infty}$ in sets of small measure.
\end{abstract}

\section{INTRODUCTION}

Suppose we are given a time-harmonic incident electric field $\mathscr{E}^{i}$ and magnetic field $\mathscr{H}^{i}$, special solutions of the time-harmonic homogeneous linear Maxwell equations of the form $\mathscr{E}^{i}=\Re\left(\mathbf{E}^{i} e^{-i \omega t}\right)$ and magnetic field $\mathscr{H}^{i}=\Re\left(\mathbf{H}^{i} e^{-i \omega t}\right)$, where $\mathbf{E}^{i} \in$ $H_{\text {loc }}^{1}\left(\mathbb{R}^{3}\right)^{3}$ and $\mathbf{H}^{i} \in H_{\text {loc }}^{1}\left(\mathbb{R}^{3}\right)^{3}$ are complex-valued solutions of the homogeneous time-harmonic Maxwell equations

$$
\begin{aligned}
& \nabla \wedge \mathbf{E}^{i}-i \omega \mu_{0} \mathbf{H}^{i}=0 \text { in } \mathbb{R}^{3}, \\
& \nabla \wedge \mathbf{H}^{i}+i \omega \varepsilon_{0} \mathbf{E}^{i}=0 \text { in } \mathbb{R}^{3},
\end{aligned}
$$

where $\mu_{0}$ and $\varepsilon_{0}$ are positive constants, representing respectively the magnetic permeability and the electric permittivity of vacuum, and $\omega \in \mathbb{R} \backslash\{0\}$. The full time-harmonic electromagnetic field $(\mathbf{E}, \mathbf{H}) \in H_{\text {loc }}\left(\operatorname{curl} ; \mathbb{R}^{3}\right)$, where for any domain $W$ we define

$$
H_{\mathrm{loc}}(\operatorname{curl} ; W):=\left\{\mathbf{u} \in L_{\mathrm{loc}}^{2}(W)^{3} \text { such that } \nabla \wedge \mathbf{u} \in L_{\mathrm{loc}}^{2}(W)^{3}\right\},
$$

satisfies Maxwell's equations

$$
\begin{aligned}
\nabla \wedge \mathbf{E}-i \omega \mu_{0} \mu(x) \mathbf{H} & =0 \text { in } \mathbb{R}^{3}, \\
\nabla \wedge \mathbf{H}+i \omega \varepsilon_{0} \varepsilon(x) \mathbf{E} & =0 \text { in } \mathbb{R}^{3},
\end{aligned}
$$

where $\varepsilon$ and $\mu$ are real matrix-valued functions in $L^{\infty}\left(\mathbb{R}^{3}\right)^{3 \times 3}$. Decomposing the full electromagnetic field into its incident part and its scattered part,

$$
\mathbf{E}^{s}:=\mathbf{E}-\mathbf{E}^{i}, \text { and } \mathbf{H}^{s}:=\mathbf{H}-\mathbf{H}^{i},
$$


we assume that the scattered field satisfies the Silver-Müller radiation condition, uniformly in all directions, that is, if $x:=r \theta$ then

$$
\lim _{r \rightarrow \infty} \sup _{\theta \in S^{2}}\left|\mathbf{H}^{s}(r \theta) \wedge r \theta-r \mathbf{E}^{s}(r \theta)\right|=0,
$$

where $S^{2}:=\left\{x \in \mathbb{R}^{3}\right.$ such that $\left.|x|=1\right\}$ denotes the unit sphere.

This paper is about the existence of a unique solution to (1.1) satisfying (1.2) and (1.3), under the following additional hypotheses on $\varepsilon$ and $\mu$. We assume that both permittivity and permeability are real symmetric, uniformly positive definite and bounded, that is, there exist $0<\alpha \leq \beta<\infty$ such that for all $\xi \in \mathbb{R}^{3}$ and almost every $x \in \mathbb{R}^{3}$,

$$
\begin{aligned}
& \alpha|\xi|^{2} \leq \varepsilon(x) \xi \cdot \xi \leq \beta|\xi|^{2}, \\
& \alpha|\xi|^{2} \leq \mu(x) \xi \cdot \xi \leq \beta|\xi|^{2} .
\end{aligned}
$$

We suppose that $\varepsilon$ and $\mu$ vary only in an open bounded domain $\Omega$, so that

$$
\varepsilon=\mu=\mathbf{I}_{3} \text { in } \Omega^{c}=\mathbb{R}^{3} \backslash \Omega,
$$

where $\mathbf{I}_{3}$ is the identity matrix in $\mathbb{R}^{3 \times 3}$. We assume that $\Omega$ is of the form

$$
\Omega=\operatorname{int}\left(\underset{i \in I}{\cup} \bar{\Omega}_{i}\right),
$$

where the sub-domains $\Omega_{i}, i \in I \subset \mathbb{N}$ are disjoint and of class $C^{0}$, and int denotes the interior. The permittivity $\varepsilon$ and the permeability $\mu$ are assumed to be piecewise $W^{1, \infty}$ with respect to the sub-domains $\Omega_{i}$, so that for each $i \in I$, there exist $\varepsilon_{i}, \mu_{i} \in W^{1, \infty}\left(\mathbb{R}^{3}\right)^{3 \times 3}$ satisfying (1.4)-(1.5) and

$$
\left\|\varepsilon_{i}\right\|_{W^{1, \infty}\left(\mathbb{R}^{3}\right)^{3 \times 3}}+\left\|\mu_{i}\right\|_{W^{1, \infty}\left(\mathbb{R}^{3}\right)^{3 \times 3}} \leq M_{i},
$$

where $M_{i}>0$ is a positive constant, such that

$$
\varepsilon(x)=\varepsilon_{i}(x) \text { and } \mu(x)=\mu_{i}(x), \quad \text { a.e. } x \in \Omega_{i} .
$$

Given a bounded set $A \subset \mathbb{R}^{3}$, we write $\mathcal{U}(A)$ as the (unique) unbounded component of $\bar{A}^{c}$.

Assumption 1. For any $J \subset I$, and $\Omega_{J}=\operatorname{int}\left(\underset{j \in J}{\cup} \bar{\Omega}_{j}\right)$, there exists $j_{0} \in J$ such that $\partial \mathcal{U}\left(\Omega_{J}\right) \cap \partial \Omega_{j_{0}}$ admits an interior point relative to $\partial \mathcal{U}\left(\Omega_{J}\right)$. In other words, there exist $j_{0} \in J$ and $x_{0} \in \partial \mathcal{U}\left(\Omega_{J}\right) \cap \partial \Omega_{j_{0}}$ such that $B\left(x_{0}, \delta\right) \cap \partial \mathcal{U}\left(\Omega_{J}\right) \subset \partial \Omega_{j_{0}}$ for some $\delta>0$.

Proposition 1. Assumption 1 holds if for all $J \subset I$, there exist $x_{J} \in \partial \mathcal{U}\left(\Omega_{J}\right)$ and $\delta_{J}>0$ such that $B\left(x_{J}, \delta_{J}\right) \cap \Omega_{j} \neq \emptyset$ for only finitely many $j \in J$. In particular, Assumption 1 holds when I is finite.

Proof. Given $J, x_{J} \in \partial \mathcal{U}\left(\Omega_{J}\right)$ and $\delta_{J}$ as in the statement of the proposition let $B_{J}=B\left(x_{J}, \delta_{J}\right)$ and let $J^{\prime}$ be the finite subset of $J$ such that $B_{J} \cap \cup_{j \in J} \Omega_{j}=$ $B_{J} \cap \underset{j \in J^{\prime}}{\cup} \Omega_{j}$. We first show that

$$
\partial \mathcal{U}\left(\Omega_{J}\right) \cap B_{J}=\cup_{j \in J}\left(\partial \mathcal{U}\left(\Omega_{J}\right) \cap B_{J}\right) \cap \partial \Omega_{j} .
$$

Indeed, let $x \in \partial \mathcal{U}\left(\Omega_{J}\right) \cap B_{J}$. Then $x \notin \underset{j \in J}{\cup} \Omega_{j}$. We claim that there exists a sequence $x_{k} \in \underset{j \in J}{\cup} \Omega_{j}$ such that $x_{k}$ tends to $x$. If not, for some $\eta>0$ sufficiently 

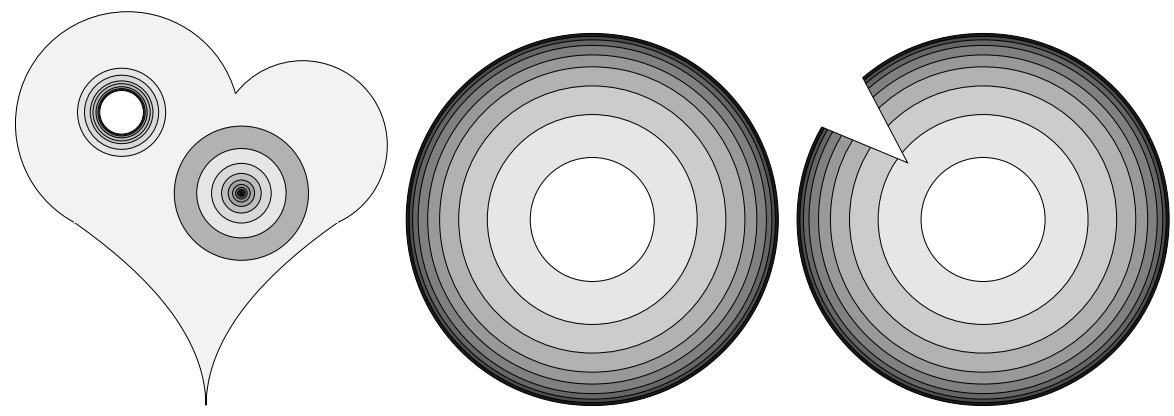

Figure 1. Left: an infinite collection of sub-domains satisfying Assumption 1. Centre: an infinite collection of sub-domains excluded by Assumption 1, Right: this collection satisfies Assumption 1 .

small, we would have $B(x, \eta) \subset B_{J}$, and $B(x, \eta) \cap \underset{j \in J}{\cup} \Omega_{j}=B(x, \eta) \cap \underset{j \in J^{\prime}}{\cup} \Omega_{j}=\emptyset$. On the other hand, there exists a sequence $y_{k} \in \mathcal{U}\left(\Omega_{J}\right)$ such that $y_{k}$ tends to $x$. But $B(x, \eta)$ is connected and contained in $\bar{\Omega}_{J}^{c}$, thus $B(x, \eta) \subset \mathcal{U}\left(\Omega_{J}\right)$. This contradiction proves the claim.

Next, we note that $\partial \mathcal{U}\left(\Omega_{J}\right)$ is closed, thus complete in the subspace topology induced by $\mathbb{R}^{3}$. Its intersection with the open ball $B_{J}$ is an open subspace of $\partial \mathcal{U}\left(\Omega_{J}\right)$ by definition of the subspace topology. It is therefore a Baire space (see e.g. [11]). If a Baire Space is a countable union of closed sets, then one of the sets has an interior point. Using the identity (1.10), we obtain that there exists $j_{0}$ such that $\partial \mathcal{U}\left(\Omega_{J}\right) \cap B_{J} \cap \partial \Omega_{j_{0}}$ admits an interior point relative to $\partial \mathcal{U}\left(\Omega_{J}\right) \cap B_{J}$, that is, there exist $j_{0} \in J, x_{0} \in \partial \mathcal{U}\left(\Omega_{J}\right) \cap B_{J}$ and $\delta>0$ such that $B\left(x_{0}, \delta\right) \cap \partial \mathcal{U}\left(\Omega_{J}\right) \cap B_{J} \subset \partial \Omega_{j_{0}}$.

Since $B_{J}$ is open, $B\left(x_{0}, \delta\right) \cap B_{J}=B\left(x_{0}, \delta\right)$ when $\delta$ is sufficiently small, and we have established that Assumption 1 holds.

An example of a collection of sub-domains excluded by Assumption 1 is a collection of concentric shells concentrating on an exterior boundary, such as

$$
\Omega_{i}=B\left(\mathbf{0}, \frac{i}{i+1}\right) \backslash \bar{B}\left(\mathbf{0}, \frac{i-1}{i}\right), \quad i=1,2,3, \ldots
$$

In such a case, $\partial \mathcal{U}(\Omega)$ is the unit sphere, which is not the boundary of any of the subsets. On the other hand, Assumption 1 allows the sub-domains $\Omega_{i}$ to concentrate at a point or near an interior boundary. In Figure 1, we represent on the left a non-Lipschitz non-simply connected domain $\Omega$ which satisfies Assumption 1. In the centre, the domain given by (1.11) excluded by Assumption 1 is shown. On the right, we sketch a domain inspired by the one described by (1.11) which satisfies Assumption 1: near the accumulating boundary, interior points can be found on the wedge-shaped slit in the domain.

\section{Main Result}

Our main result is the following theorem.

Theorem 2. Assume that (1.4)-(1.9) and Assumption 1 hold. If for a given $\omega \neq 0$, $\mathbf{E} \in H_{\text {loc }}\left(\operatorname{curl} ; \mathbb{R}^{3}\right)$ and $\mathbf{H} \in H_{\text {loc }}\left(\operatorname{curl} ; \mathbb{R}^{3}\right)$ are solutions of (1.1) -(1.3) corresponding to $\mathbf{E}^{i}=0$ and $\mathbf{H}^{i}=0$, then $\mathbf{E}=\mathbf{H}=0$. 
There is a very long history concerning this problem, under various assumptions on the coefficients, see e.g. [1, 2, 3, 6, 17, 10, 13, 12, 14, 16, and the references therein. The improvement provided by the result in this work is that we assume that $\varepsilon, \mu$ are matrix-valued functions and that the sub-domains $\Omega_{i}$ are only of class $C^{0}$. We do not assume that the sub-domains are Lipschitz as assumed for example in [6] for the isotropic (scalar) case. The authors are not aware of the existence of a general uniqueness result for the above problem when the coefficients are just $C^{0, \alpha}$ Hölder continuous, with $\alpha<1$. For general elliptic equations, counter-examples to unique continuation, the main technique for proving uniqueness, are known in that case, see [8]. We remind the reader of the definition of a domain of class $C^{0}$.

Definition 3. $A$ bounded domain $\Omega$ of $\mathbb{R}^{3}$ is of class $C^{0}$ if for any point $x_{0}$ on the boundary $\partial \Omega$, there exists a ball $B\left(x_{0}, \delta\right)$ and an orthogonal coordinate system $\left(x_{1}, x_{2}, x_{3}\right)$ with origin at $x_{0}$ such that there exists a continuous function $f: C^{0}\left(\mathbb{R}^{2} ; \mathbb{R}\right)$ that satisfies

$$
\Omega \cap B\left(x_{0}, \delta\right)=\left\{x \in B\left(x_{0}, \delta\right): x_{3}>f\left(x_{1}, x_{2}\right)\right\} .
$$

We define $B_{0}$ as the smallest open ball containing $\Omega$. Note that the uniqueness of the solution outside $B_{0}$ is well known, due to the so-called Rellich's Lemma, see e.g. [2].

Lemma 4 (Rellich's Lemma). If for a fixed $\omega, \mathbf{E} \in H_{\mathrm{loc}}\left(\operatorname{curl} ; \mathbb{R}^{3}\right)$ and $\mathbf{H} \in$ $H_{\mathrm{loc}}\left(\mathrm{curl} ; \mathbb{R}^{3}\right)$ are solutions of (1.1)-(1.3) corresponding to $\mathbf{E}^{i}=0$ and $\mathbf{H}^{i}=0$, then $\mathbf{E}=\mathbf{H}=0$ in $\bar{B}_{0}^{c}$.

Our proof relies on a recent unique continuation result 13 proved for globally $W^{1, \infty}$ regular coefficients.

Theorem 5 ([13]). Let $V$ be a connected open set in $\mathbb{R}^{3}$. Assume that $\varepsilon$ and $\mu$ are two real symmetric matrix valued functions in $V$ satisfying (1.4)-(1.5), and

$$
\|\varepsilon\|_{W^{1, \infty}(V)^{3 \times 3}}+\|\mu\|_{W^{1, \infty}(V)^{3 \times 3}} \leq M,
$$

for some constant $M>0$. Suppose $(\mathbf{E}, \mathbf{H}) \in\left(L_{\mathrm{loc}}^{2}(V)\right)^{2}$ satisfy

$$
\begin{aligned}
& \nabla \wedge \mathbf{E}-i \omega \mu_{0} \mu(x) \mathbf{H}=0 \text { in } V, \\
& \nabla \wedge \mathbf{H}+i \omega \varepsilon_{0} \varepsilon(x) \mathbf{E}=0 \text { in } V .
\end{aligned}
$$

Then, there exist $s>0$ independent of $V, \mathbf{E}$ and $\mathbf{H}$, such that if for some $x_{0} \in V$, and for all $N \in \mathbb{N}$ and all $\delta>0$ sufficiently small,

$$
\int_{B\left(x_{0}, \delta\right)}\left(|\mathbf{E}|^{2}+|\mathbf{H}|^{2}\right) d x \leq C_{N} \exp \left(-N \delta^{-s}\right)
$$

for some constant $C_{N}>0$, then $\mathbf{E}=\mathbf{H}=0$ in $V$.

The proof of Theorem 2 consists of three steps. The first two steps are given by the two propositions below.

Proposition 6. Under the hypothesis of Theorem 2 , suppose that $A \subset \mathbb{R}^{3}$ is a bounded open set and that for almost every $x \in \mathcal{U}(A)$ either $\varepsilon(x)=\mu(x)=\mathbf{I}_{3}$ or $\mathbf{E}(x)=\mathbf{H}(x)=0$. Then $\mathbf{E}=\mathbf{H}=0$ in $\mathcal{U}(A)$. 


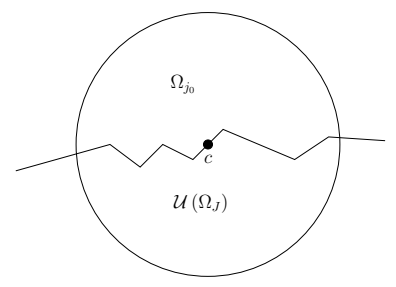

FiguRE 2. A ball centred at an interior point on the boundary of $\mathcal{U}\left(\Omega_{J}\right)$.

Proof. For any $\mathbf{v}, \mathbf{w} \in L^{2}(\mathcal{U}(A))^{2}$, we have

$$
\begin{gathered}
\int_{\mathcal{U}(A)} \nabla \wedge \mathbf{E} \cdot \mathbf{v} d x-i \omega \mu_{0} \int_{\mathcal{U}(A)} \mu(x) \mathbf{H} \cdot \mathbf{v} d x=0, \\
\int_{\mathcal{U}(A)} \nabla \wedge \mathbf{H} \cdot \mathbf{w} d x+i \omega \varepsilon_{0} \int_{\mathcal{U}(A)} \epsilon(x) \mathbf{E} \cdot \mathbf{w} d x=0,
\end{gathered}
$$

where the integrals (2.1) and (2.2) are well defined by Rellich's Lemma 4. Since for almost every $x$ in $\mathcal{U}(A)$, either $\varepsilon(x)=\mu(x)=\mathbf{I}_{3}$ or $\mathbf{E}=\mathbf{H}=0$, the solutions of the system (2.1)-(2.2) can be written also in the form

$$
\begin{aligned}
& \int_{\mathcal{U}(A)} \nabla \wedge \mathbf{E} \cdot \mathbf{v} d x-i \omega \mu_{0} \int_{\mathcal{U}(A)} \mathbf{H} \cdot \mathbf{v} d x=0, \\
& \int_{\mathcal{U}(A)} \nabla \wedge \mathbf{H} \cdot \mathbf{w} d x+i \omega \varepsilon_{0} \int_{\mathcal{U}(A)} \mathbf{E} \cdot \mathbf{w} d x=0,
\end{aligned}
$$

which is the weak formulation of

$$
\begin{aligned}
& \nabla \wedge \mathbf{E}-i \omega \mu_{0} \mathbf{H}=0 \text { in } \mathcal{U}(A), \\
& \nabla \wedge \mathbf{H}+i \omega \varepsilon_{0} \mathbf{E}=0 \text { in } \mathcal{U}(A) .
\end{aligned}
$$

Next, since $A$ is bounded, thanks to Rellich's Lemma 4, $\mathbf{E}=\mathbf{H}=0$ in $\mathcal{U}(A) \cap$ $\left(\mathbb{R}^{3} \backslash \bar{B}(R)\right)$, for $R$ large enough. In particular, $\mathbf{E}$ and $\mathbf{H}$ vanish in a ball contained in $\mathcal{U}(A)$, which is open and connected, and the conclusion follows from Theorem 5 , applied with $\varepsilon(x)=\mu(x)=\mathbf{I}_{3}$, which in this case reduces to a well known result concerning the Helmholtz equation.

\section{Proposition 7. Let}

$$
J:=\left\{i \in I:|\mathbf{E}(x)|^{2}+|\mathbf{H}(x)|^{2}>0 \text { on a set of positive measure in } \Omega_{i}\right\} .
$$

Then $J=\emptyset$.

Proof. Suppose for contradiction that $J$ is nonempty. Then, by Assumption 1 there exists $x_{0} \in \partial \mathcal{U}\left(\Omega_{J}\right) \cap \partial \Omega_{j_{0}}$ such that $B\left(x_{0}, \delta\right) \cap \partial \mathcal{U}\left(\Omega_{J}\right) \subset \partial \Omega_{j_{0}}$ for some $j_{0} \in J$ and $\delta>0$. To simplify notation, set $j_{0}=1$.

Let us show that there exist a point $c$ on $\partial \mathcal{U}\left(\Omega_{J}\right) \cap \partial \Omega_{1}$ and a radius $\tilde{\delta}>0$ such that

$$
\Omega_{J} \cap B(c, \tilde{\delta})=\Omega_{1} \cap B(c, \tilde{\delta}) .
$$

Figure 2 sketches the configuration we have at hand around $c$.

Since $\Omega_{1}$ has a $C^{0}$ boundary, for some (smaller) $\delta>0$ there exists a continuous map $f$ and a suitable orientation of axes such that $B\left(x_{0}, \delta\right) \cap \partial \Omega_{J} \subset \partial \Omega_{1}$ and

$$
\Omega_{1} \cap B\left(x_{0}, \delta\right)=\left\{x \in B\left(x_{0}, \delta\right): x_{3}>f\left(x_{1}, x_{2}\right)\right\} .
$$


This alone does not prove our claim, since $B\left(x_{0}, \delta\right)$ could still intersect $\Omega_{J}$ when $x_{3} \leq f\left(x_{1}, x_{2}\right)$. Since $x_{0} \in \partial \mathcal{U}\left(\Omega_{J}\right)$, there exists a sequence $\left\{y_{j}\right\} \subset \mathcal{U}\left(\Omega_{J}\right) \cap B\left(x_{0}, \delta\right)$ such that $y_{j}$ tends to $x_{0}$. Consider for a fixed and sufficiently large $j$ the line segment $\left\{y_{j}+t e_{3}, t \geq 0\right\}$, and let $\tau>0$ be the least value of $t$ such that $y_{j}+t e_{3} \in \partial \Omega_{J}$. Then, $y_{j}+t e_{3} \notin \bar{\Omega}_{J}$, for $t<\tau$, and $y_{j}+\tau e_{3} \in \partial \Omega_{1}$. Hence $y_{j}+\tau e_{3} \notin \underset{k \in J, k>1}{\cup} \Omega_{k}$.

Since the sets $\Omega_{k}$ are disjoint, the line segment does not intersect $\underset{k \in J, k>1}{\cup} \Omega_{k}$ in $B\left(x_{0}, \delta\right)$. The same argument applies to any line segment $\left\{z+t e_{3}, t \geq 0\right\}$ for $z$ sufficiently close to $y_{j}$. Introducing $c=y_{j}+\tau e_{3}$ we have established that there exists a ball $B(c, \tilde{\delta})$ such that $\Omega_{1} \cap B(c, \tilde{\delta})=\Omega_{J} \cap B(c, \tilde{\delta})$, which is (2.3).

Now, thanks to Proposition [6, and noting that (by Fubini's Theorem) each $\partial \Omega_{i}$ is of measure zero, $\mathbf{E}=\mathbf{H}=0$ almost everywhere in $\mathcal{U}\left(\Omega_{J}\right)$. Thus, for almost every $x \in B(c, \tilde{\delta})$, either $\mathbf{E}=\mathbf{H}=0$ or $\varepsilon(x)=\varepsilon_{1}(x)$, and $\mu(x)=\mu_{1}(x)$. Considering the weak formulation of Maxwell's equations, and arguing as in the proof of Proposition [6] we note that $\mathbf{E}$ and $\mathbf{H}$ are weak solutions of

$$
\begin{aligned}
& \nabla \wedge \mathbf{E}-i \omega \mu_{0} \mu_{1}(x) \mathbf{H}=0 \text { in } B(c, \tilde{\delta}), \\
& \nabla \wedge \mathbf{H}+i \omega \varepsilon_{0} \varepsilon_{1}(x) \mathbf{E}=0 \text { in } B(c, \tilde{\delta}),
\end{aligned}
$$

and vanish on the connected non-empty open set $B(c, \tilde{\delta}) \cap\left\{x_{3}<f\left(x_{1}, x_{2}\right)\right\}$. Since $\epsilon_{1}$ and $\mu_{1}$ satisfy (1.8), that is,

$$
\left\|\varepsilon_{1}\right\|_{W^{1, \infty}\left(\mathbb{R}^{3}\right)^{3 \times 3}}+\left\|\mu_{1}\right\|_{W^{1, \infty}\left(\mathbb{R}^{3}\right)^{3 \times 3}} \leq M_{1},
$$

Theorem 5 shows that $\mathbf{E}=\mathbf{H}=0$ in $B(c, \tilde{\delta})$. This in turn shows that $\mathbf{E}$ and $\mathbf{H}$ vanish on a ball inside $\Omega_{1}$, and applying Theorem 5 in $\Omega_{1}$ we obtain $\mathbf{E}=\mathbf{H}=0$ almost everywhere in $\Omega_{1}$. This contradiction concludes the proof.

We now turn to the final step. We have obtained that $\mathbf{E}=\mathbf{H}=0$ almost everywhere in $\Omega$, and therefore either $\mathbf{E}=\mathbf{H}=0$ or $\varepsilon(x)=\mu(x)=\mathbf{I}_{3}$ almost everywhere in $\mathbb{R}^{3}$. Arguing as above, we deduce that $(\mathbf{E}, \mathbf{H})$ is a weak solution of (1.1) with $\varepsilon(x)=\mu(x)=\mathbf{I}_{3}$ everywhere and the conclusion of Theorem 2 follows from Rellich's Lemma.

\section{The CASE OF A Medium With DEFECTS}

We extend our result to the case when defects of small measure are allowed in the medium. One application is to liquid crystals (see [15] for more details). Namely, we assume that the permittivity and permeability are of the form

$$
\begin{aligned}
\varepsilon_{D} & =\left(1-\mathbf{1}_{D}\right) \varepsilon+\mathbf{1}_{D} \tilde{\varepsilon}, \\
\mu_{D} & =\left(1-\mathbf{1}_{D}\right) \mu+\mathbf{1}_{D} \tilde{\mu},
\end{aligned}
$$

where $\varepsilon$ and $\mu$ satisfy (1.4)-(1.9), $\mathbf{1}_{D}$ is the indicator function of a measurable bounded set $D$, such that

$$
D \subset \cup \Omega_{i \in I} \Omega_{i}, \quad \overline{D \cap \Omega_{i}} \subset \Omega_{i} \text { and } \Omega_{i} \backslash \overline{D \cap \Omega_{i}} \text { is connected for each } i \in I,
$$

and $\tilde{\epsilon}$ and $\tilde{\mu}$ are real symmetric positive definite matrices in $L^{\infty}\left(\mathbb{R}^{3}\right)^{3 \times 3}$ satisfying (1.4) $-(1.5)$. 
Theorem 8. Suppose that the electric and magnetic fields $\mathbf{E}_{D} \in H_{\mathrm{loc}}\left(\mathrm{curl} ; \mathbb{R}^{3}\right)$ and $\mathbf{H}_{D} \in H_{\text {loc }}\left(\mathrm{curl} ; \mathbb{R}^{3}\right)$ are solutions of

$$
\begin{gathered}
\nabla \wedge \mathbf{E}_{D}-i \omega \mu_{0} \mu_{D}(x) \mathbf{H}_{D}=0 \text { in } \mathbb{R}^{3}, \\
\nabla \wedge \mathbf{H}_{D}+i \omega \varepsilon_{0} \varepsilon_{D}(x) \mathbf{E}_{D}=0 \text { in } \mathbb{R}^{3},
\end{gathered}
$$

together with the Silver-Müller radiation condition (1.3), and that $\varepsilon_{D}$ and $\mu_{D}$ are given by (3.1), with $D$ satisfying (3.2). Suppose Assumption 1 holds. Then, there exists a constant $d_{0}>0$ depending only on the measure $\left|B_{0}\right|$ of $B_{0},|\omega|$ and the lower and upper bounds $\alpha$ and $\beta$ given in (1.4)-(1.5) such that if the measure of $D$ satisfies $|D|<d_{0}$, then $\mathbf{E}_{D}=\mathbf{H}_{D}=0$ almost everywhere.

To prove Theorem 8 , we use the following variant of Theorem 2

Proposition 9. Under the same assumptions as Theorem[2, and assuming that (3.2) holds,

$$
\operatorname{supp} \mathbf{H}_{D} \cup \operatorname{supp} \mathbf{E}_{D} \subset \bar{D} .
$$

Proof. The proof follows from that of Theorem 2 , since by assumption for each $i \in I, \overline{D \cap \Omega_{i}} \subset \Omega_{i}$, and the boundary of $\Omega \backslash \Omega_{i}$ is unaltered by the defects.

Proof of Theorem 8. Since (3.3) admits a weak formulation, arguing as before we see using Proposition 9 that $\mathbf{E}_{D} \in H\left(\operatorname{curl} ; B_{0}\right)$ and $\mathbf{H}_{D} \in H\left(\operatorname{curl} ; B_{0}\right)$ have compact support in $B_{0}$ and are also solutions of

$$
\begin{gathered}
\nabla \wedge \mathbf{E}_{D}-i \omega \mu_{0} \hat{\mu} \mathbf{H}_{D}=0 \text { in } B_{0}, \\
\nabla \wedge \mathbf{H}_{D}+i \omega \varepsilon_{0} \hat{\epsilon} \mathbf{E}_{D}=0 \text { in } B_{0},
\end{gathered}
$$

where $\hat{\varepsilon}=\mathbf{I}_{3}+\mathbf{1}_{D}\left(\tilde{\varepsilon}-\mathbf{I}_{3}\right)$, and $\hat{\mu}=\mathbf{I}_{3}+\mathbf{1}_{D}\left(\tilde{\mu}-\mathbf{I}_{3}\right)$. Note that $i \omega \mu_{0} \hat{\mu} \mathbf{H}_{D}$ has compact support and is divergence free. Thus the Helmholtz decomposition (see e.g. [4, 5, 9]) of $i \omega \mu_{0} \hat{\mu} \mathbf{H}_{D}$ shows there exists a unique $\mathbf{A}_{H} \in H^{1}\left(B_{0}\right)^{3}$ such that $\mathbf{A}_{H} \cdot \nu=0$, on $\partial B_{0}, \operatorname{div}\left(\mathbf{A}_{H}\right)=0$ and such that $i \omega \mu_{0} \hat{\mu} \mathbf{H}_{D}=\nabla \wedge \mathbf{A}_{H}$. Furthermore, $\mathbf{A}_{H}$ satisfies

$$
\begin{aligned}
\left\|\nabla \mathbf{A}_{H}\right\|_{L^{2}\left(B_{0}\right)^{3 \times 3}} & \leq C\left(\left\|\nabla \wedge \mathbf{A}_{H}\right\|_{L^{2}\left(B_{0}\right)^{3}}+\left\|\mathbf{A}_{H}\right\|_{L^{2}\left(B_{0}\right)^{3}}\right) \\
\text { and }\left\|\mathbf{A}_{H}\right\|_{L^{2}\left(B_{0}\right)^{3}} & \leq C\left|B_{0}\right|^{1 / 3}\left\|\nabla \wedge \mathbf{A}_{H}\right\|_{L^{2}\left(B_{0}\right)^{3}},
\end{aligned}
$$

where $C$ is a universal constant. Altogether this yields

$$
\left\|\nabla \mathbf{A}_{H}\right\|_{L^{2}\left(B_{0}\right)^{3 \times 3}} \leq C \beta \mu_{0}|\omega|\left(\left|B_{0}\right|+1\right)^{1 / 3}\left\|\mathbf{H}_{D}\right\|_{L^{2}\left(B_{0}\right)^{3}} .
$$

Since $\mathbf{E}_{D}-\mathbf{A}_{H}$ is curl free, we deduce that there exists $p \in H^{1}\left(B_{0}\right)$ such that $\mathbf{E}_{D}=\mathbf{A}_{H}+\nabla p$, and $p$ is uniquely defined by setting $\int_{B_{0}} p d x=0$. Noticing that $\hat{\varepsilon} \mathbf{E}_{D}$ is divergence free, and $\hat{\varepsilon}-\mathbf{I}_{3}$ is compactly supported in $B_{0}$ we have that $p$ is the solution of

$$
\begin{aligned}
\operatorname{div}(\hat{\varepsilon} \nabla p) & =-\operatorname{div}\left(\hat{\varepsilon} \mathbf{A}_{H}\right) \text { in } B_{0}, \\
\nabla p \cdot n & =0 \text { on } \partial B_{0}, \\
\int_{B_{0}} p d x & =0 .
\end{aligned}
$$

Since $\mathbf{A}_{H}$ is divergence free, the right-hand side becomes

$$
-\operatorname{div}\left(\hat{\varepsilon} \mathbf{A}_{H}\right)=-\operatorname{div}\left(\mathbf{1}_{D}\left(\tilde{\varepsilon}-\mathbf{I}_{3}\right) \mathbf{A}_{H}\right) .
$$


To proceed, we compute using the Cauchy-Schwarz inequality the following bound

$$
\begin{aligned}
\alpha\|\nabla p\|_{L^{2}\left(B_{0}\right)^{3}}^{2} \leq \int_{B_{0}} \hat{\epsilon} \nabla p \cdot \nabla p d x & =-\int_{B_{0}} \mathbf{1}_{D}\left(\tilde{\varepsilon}-\mathbf{I}_{3}\right) \mathbf{A}_{H} \cdot \nabla p d x \\
& \leq(\beta+1)\left\|\mathbf{A}_{H}\right\|_{L^{2}(D)^{3}}\|\nabla p\|_{L^{2}\left(B_{0}\right)^{3}},
\end{aligned}
$$

and we have obtained that

$$
\|\nabla p\|_{L^{2}\left(B_{0}\right)^{3}} \leq \frac{\beta+1}{\alpha}\left\|\mathbf{A}_{H}\right\|_{L^{2}(D)^{3}} .
$$

Next note using Proposition9 that

$$
\left\|\mathbf{E}_{D}\right\|_{L^{2}\left(B_{0}\right)^{3}}=\left\|\mathbf{E}_{D}\right\|_{L^{2}(D)^{3}} \leq\|\nabla p\|_{L^{2}\left(B_{0}\right)^{3}}+\left\|\mathbf{A}_{H}\right\|_{L^{2}(D)^{3}} \leq \frac{2 \beta+1}{\alpha}\left\|\mathbf{A}_{H}\right\|_{L^{2}(D)^{3}} .
$$

The Sobolev-Gagliardo-Nirenberg inequality in $B_{0}$ shows that

$$
\left\|\mathbf{A}_{H}\right\|_{L^{6}\left(B_{0}\right)^{3}} \leq C\left(\left|B_{0}\right|+1\right)^{1 / 3}\left\|\mathbf{A}_{H}\right\|_{H^{1}\left(B_{0}\right)^{3}},
$$

where $C$ is a universal constant. Therefore, using Hölder's inequality, together with the Poincaré-Friedrichs estimate (3.4), we have

$$
\left\|\mathbf{A}_{H}\right\|_{L^{2}(D)^{3}} \leq|D|^{\frac{1}{3}}\left\|\mathbf{A}_{H}\right\|_{L^{6}\left(B_{0}\right)^{3}} \leq C \beta\left(\left|B_{0}\right|+1\right)^{2 / 3} \mu_{0}|\omega||D|^{\frac{1}{3}}\left\|\mathbf{H}_{D}\right\|_{L^{2}\left(B_{0}\right)^{3}} .
$$

Altogether we have obtained

$$
\left\|\mathbf{E}_{D}\right\|_{L^{2}\left(B_{0}\right)^{3}} \leq C \frac{\beta(\beta+1)}{\alpha}\left(\left|B_{0}\right|+1\right)^{2 / 3} \mu_{0}|\omega||D|^{\frac{1}{3}}\left\|\mathbf{H}_{D}\right\|_{L^{2}\left(B_{0}\right)^{3}} .
$$

Repeating the same argument, but starting with $\mathbf{H}_{D}$, we obtain also

$$
\left\|\mathbf{H}_{D}\right\|_{L^{2}\left(B_{0}\right)^{3}} \leq C \frac{\beta(\beta+1)}{\alpha}\left(\left|B_{0}\right|+1\right)^{2 / 3} \varepsilon_{0}|\omega||D|^{\frac{1}{3}}\left\|\mathbf{E}_{D}\right\|_{L^{2}\left(B_{0}\right)^{3}} .
$$

The inequalities (3.5) and (3.6) imply that $\mathbf{H}_{D}=\mathbf{E}_{D}=0$ when

$$
|D|<d_{0}:=C \frac{\alpha^{3}}{\beta^{3}(\beta+1)^{3}\left(\left|B_{0}\right|+1\right)^{2}\left(\sqrt{\varepsilon_{0} \mu_{0}}|\omega|\right)^{3}},
$$

where $C$ is a universal constant.

Remark 10. The dependence of the threshold constant $d_{0}$ given by (3.7) on $|\omega|$ and $\left|B_{0}\right|$ shows that for a permeability $\mu$ and a permittivity $\varepsilon$ satisfying (1.4), (1.5) and (1.6) only, uniqueness for Maxwell's equations holds provided, if $\omega$ is fixed, the domain $\Omega$ is of small measure and bounded diameter, or, for a given $\Omega$, when the absolute value of the frequency $|\omega|$ is sufficiently small. In such cases, the whole domain $\Omega$ can be taken as a defect $D$ (and a fictitious ball containing $D$ plays the role of $\Omega$ ). We do not claim that the dependence of $d_{0}$ in terms of $|\omega|$ or $\left|B_{0}\right|$ in (3.7) is optimal. In contrast, Theorem 2 requires additional regularity assumptions on $\mu$ and $\varepsilon$, but does not depend on the frequency or the size of the domain.

\section{ACKNOWLEDGEMENTS}

The authors were supported by EPSRC Grant EP/E010288/1 and by the EPSRC Science and Innovation award to the Oxford Centre for Nonlinear PDE (EP/E035027/1). 


\section{REFERENCES}

[1] F. Cakoni and D. Colton. A uniqueness theorem for an inverse electromagnetic scattering problem in inhomogeneous anisotropic media. Proc. Edinb. Math. Soc. (2), 46(2):293-314, 2003.

[2] D. Colton and R. Kress. Inverse acoustic and electromagnetic scattering theory, volume 93 of Applied Mathematical Sciences. Springer-Verlag, Berlin, second edition, 1998.

[3] M. M. Eller and M. Yamamoto. A Carleman inequality for the stationary anisotropic Maxwell system. J. Math. Pures Appl. (9), 86(6):449-462, 2006.

[4] K. O. Friedrichs. Differential forms on Riemannian manifolds. Comm. Pure Appl. Math., 8:551-590, 1955.

[5] V. Girault and P.-A. Raviart. Finite element approximation of the Navier-Stokes equations, volume 749 of Lecture Notes in Mathematics. Springer-Verlag, Berlin, 1979.

[6] C. Hazard and M. Lenoir. On the solution of time-harmonic scattering problems for Maxwell's equations. SIAM J. Math. Anal., 27(6):1597-1630, 1996.

[7] R. Leis. Über die eindeutige Fortsetzbarkeit der Lösungen der Maxwellschen Gleichungen in anisotropen inhomogenen Medien. Bul. Inst. Politehn. Iaşi (N.S.), 14 (18)(fasc. 3-4):119-124, 1968.

[8] K. Miller. Nonunique continuation for uniformly parabolic and elliptic equations in self-adjoint divergence form with Hölder continuous coefficients. Arch. Rational Mech. Anal., 54:105-117, 1974.

[9] Peter Monk. Finite element methods for Maxwell's equations. Numerical Mathematics and Scientific Computation. Oxford University Press, New York, 2003.

[10] C. Müller. Foundations of the mathematical theory of electromagnetic waves. Revised and enlarged translation from the German. Die Grundlehren der mathematischen Wissenschaften, Band 155. Springer-Verlag, New York, 1969.

[11] J. R. Munkres. Topology: a first course. Prentice-Hall Inc., Englewood Cliffs, N.J., 1975.

[12] J.-C. Nédélec. Acoustic and electromagnetic equations, volume 144 of Applied Mathematical Sciences. Springer-Verlag, New York, 2001. Integral representations for harmonic problems.

[13] T. Nguyen and J.-N. Wang. Quantitative Uniqueness Estimate for the Maxwell System with Lipschitz Anisotropic Media. Proc. Amer. Math. Soc., 140(2):595-605, 2011.

[14] T. Ōkaji. Strong unique continuation property for time harmonic Maxwell equations. J. Math. Soc. Japan, 54(1):89-122, 2002.

[15] B. Tsering Xiao. Electromagnetic inverse problems for nematic liquid crystals. PhD thesis, University of Oxford, 2011.

[16] V. Vogelsang. On the strong unique continuation principle for inequalities of Maxwell type. Math. Ann., 289(2):285-295, 1991.

Mathematical Institute, 24-29 St Giles, OXFORD OX1 3LB, UK 